\title{
Factors Associated with Doses of Mood Stabilizers in Real-world Outpatients with Bipolar Disorder
}

\author{
Norio Yasui-Furukori ${ }^{1,2}$, Naoto Adachi ${ }^{3}$, Yukihisa Kubota ${ }^{3}$, Takaharu Azekawa ${ }^{3}$, Eiichiro Goto ${ }^{3}$, Koji Edagawa ${ }^{3}$, \\ Eiichi Katsumoto ${ }^{3}$, Seiji Hongo ${ }^{3}$, Hitoshi Ueda ${ }^{3}$, Kazuhira Miki ${ }^{3}$, Masaki Kato ${ }^{2,4}$, Reiji Yoshimura ${ }^{2,5}$, \\ Atsuo Nakagawa ${ }^{2,6}$, Toshiaki Kikuchi ${ }^{2,6}$, Takashi Tsuboi ${ }^{2,7}$, Koichiro Watanabe ${ }^{2,7}$, Kazutaka Shimoda ${ }^{1}$ \\ ${ }^{1}$ Department of Psychiatry, Dokkyo Medical University, School of Medicine, Tochigi, ${ }^{2}$ The Japanese Society of Clinical Neuropsychopharmacology, \\ ${ }^{3}$ The Japanese Association of Neuro-Psychiatric Clinics, Tokyo, ${ }^{4}$ Department of Neuropsychiatry, Kansai Medical University, Hirakata, ${ }^{5}$ Department \\ of Psychiatry, University of Occupational and Environmental Health, Kitakyushu, ${ }^{6}$ Department of Neuropsychiatry, Keio University School of \\ Medicine, Minato, ${ }^{7}$ Department of Neuropsychiatry, Kyorin University School of Medicine, Mitaka, Japan
}

Objective: Several evidence-based practice guidelines have been developed to better treat bipolar disorder. However, the articles cited in these guidelines were based on clinical or basic studies with specific conditional settings and were not sufficiently based on real-world clinical practice. In particular, there was little information on the doses of mood stabilizers.

Methods: The MUlticenter treatment SUrvey on Blpolar disorder in Japanese psychiatric clinics (MUSUBI) is a study conducted to accumulate evidence on the real-world practical treatment of bipolar disorder. The questionnaire included patient characteristics such as comorbidities, mental status, treatment period, Global Assessment of Functioning (GAF) score, and details of pharmacological treatment.

Results: Most patients received mood stabilizers such as lithium $(n=1,317)$, valproic acid $(n=808)$, carbamazepine $(n=136)$, and lamotrigine $(n=665)$. The dose of lithium was correlated with age, body weight, number of episodes, depression and GAF. The dose of valproic acid was correlated with body weight, number of episodes, presence of a rapid cycle and GAF. The dose of carbamazepine was correlated with age, mania, and the presence of a rapid cycle. The dose of lamotrigine was correlated with the number of episodes, depression, mania, psychotic features, and the presence of a rapid cycle. Doses of coadministered mood stabilizers were significantly correlated, except for the combination of valproic acid and lamotrigine.

Conclusion: The dose of mood stabilizers was selectively administered based on several factors, such as age, body composition, current mood status and functioning. Further prospective studies are required to confirm these findings.

KEY WORDS: Bipolar disorder; Mood stabilizers; Nationwide study; Real world; Dose.

\section{INTRODUCTION}

For the treatment of bipolar disorders, mood stabilizers are still categorized as a first-line treatment for manic and depressive episodes and the maintenance phase [1]. A previous network meta-analysis demonstrated that lithium, valproic acid (VPA), and lamotrigine (LTG) but not carbamazepine (CBZ) were more effective than placebo

Received: May 20, 2020 / Accepted: June 5, 2020

Address for correspondence: Norio Yasui-Furukori

Department of Psychiatry, Dokkoyo Medical University, School of Medicine, Mibu, Shimotsuga, Tochigi 321-0293, Japan

E-mail: furukori@dokkyomed.ac.jp

ORCID: https://orcid.org/0000-0002-4414-3770 and that the tolerability of CBZ was inferior to that of placebo [2]. However, the findings of this study are difficult to apply in the context of real-world clinical practice. The International College of Neuropsychopharmacology Treatment Guidelines for Bipolar Disorder (CINP-BD) has recently recommended that monotherapy with aripiprazole, asenapine, cariprazine, paliperidone, quetiapine, risperidone or VPA is a first-line treatment for manic patients [3]. Monotherapy with quetiapine, lurasidone or olanzapine-fluoxetine combination is the first-line treatment for depressive patients, and monotherapy with lithium, aripiprazole, olanzapine, paliperidone, quetiapine, risperidone or risperidone long-acting injectables was a

(c) This is an Open-Access article distributed under the terms of the Creative Commons Attribution Non-Commercial License (http://creativecommons.org/licenses/by-nc/4.0) which permits unrestricted non-commercial use, distribution, and reproduction in any medium, provided the original work is properly cited. 
first-line treatment in maintenance therapy [3]. In addition, the Canadian Network for Mood and Anxiety Treatments (CANMAT 2018) has recently suggested that monotherapy with lithium or divalproex is recommended as a first-line treatment for acute mania [4]. Quetiapine and lurasidone monotherapy are recommended as first-line options for bipolar depression [4]. Lithium, LTG and divalproex are recommended options for the maintenance phase [4]. The Korean Medication Algorithm Project for Bipolar Disorder (KMAP-BP) [5], which was the most recent guideline for bipolar disorder, suggested that the preferred first-step strategies were VPA, lithium, olanzapine and quetiapine for acute mania; lithium, VPA, LTG, aripiprazole, and quetiapine for depression; and a combination of mood stabilizers and atypical antipsychotics for maintenance. However, there is little information on adequate doses of mood stabilizers for the treatment of bipolar disorders, although the therapeutic reference ranges are $0.5-1.2 \mathrm{mmol} / \mathrm{L}$ for lithium, $50-100 \mu \mathrm{g} / \mathrm{ml}$ for VPA, $4-10 \mu \mathrm{g} / \mathrm{ml}$ for CBZ and $1-6 \mu \mathrm{g} / \mathrm{ml}$ for LTG [6].

A previous study suggested that the doses of mood stabilizers tend to be adjusted based on clinical response, including adverse effects. There were negative associations between serum levels of VPA and short-term delayed recall and working memory. A positive association was suggested between the serum level of lithium and working memory [7]. Although therapeutic drug monitoring (TDM) applications for neuropsychiatric agents aim to assist clinicians in enhancing the safety and efficacy of treatment, most patients do not apply for TDM in the treatment of bipolar disorders in Japan [8]. Most side effects can be transient or dose related and can be managed by optimizing drug doses to the lowest effective dose [9]. In addition, the optimal dose of mood stabilizers might have been influenced by other clinical conditions, such as age, sex, psychiatric features, comorbid symptoms and other mood stabilizers.

More than $90 \%$ of all patients with mood disorders in Japan are outpatients, 30\% of whom are treated at the clinics of Japanese Association of Neuro-Psychiatric Clinics members [10]. Therefore, we aimed to search for potential factors associated with the optimal doses of mood stabilizers in stable outpatients in good practical situations.

\section{METHODS}

\section{Study Design and Subjects}

Joint research (the MUlticenter treatment SUrvey on BIpolar disorder in Japanese psychiatric clinics, MUSUBI) was conducted between the Japanese Association of Neuro-Psychiatric Clinics and the Japanese Society of Clinical Neuropsychopharmacology to accumulate evidence on the real-world practical treatment of bipolar disorder in Japan. The method and design of MUSUBI has been described previously [11]. The MUSUBI is a crosssectional study in which a questionnaire was administered at 176 outpatient clinics belonging to the Japanese Association of Neuro-Psychiatric Clinics from September to October 2016 [11]. Patients diagnosed with bipolar disorder based on the International Classification of Diseases, 10th edition criteria (World Health Organization, 1992) and treated at these clinics were included in this study.

This study protocol was reviewed and approved by the institutional review board of the ethics committee of the Japanese Association of Neuro-Psychiatric Clinics (ID. 20160822). This study was conducted in accordance with the Declaration of Helsinki and the Japanese Ethical Guidelines for Medical and Health Research Involving Human Subjects. Prior to the initiation of the study, the study protocol was reviewed and approved by the institutional review board of the ethics committee of the Japanese Association of Neuro-Psychiatric Clinics. Since this was a retrospective medical record survey, the informed consent requirement was exempted, but we instead released information on this research so that patients were free to opt out [11].

\section{Study Procedures}

Clinical psychiatrists were asked to complete a questionnaire about patients with bipolar disorder by performing a retrospective medical record survey. The questionnaire included patient characteristics (age, sex, height, weight, academic background, mental status), treatment period, Global Assessment of Functioning (GAF) score, and details of pharmacological treatment. We mailed 20 copies of this questionnaire to each outpatient clinic and analyzed the responses. This study focused on antidepressant prescriptions for bipolar disorder patients and their profiles. 
Table 1. Characteristics of subjects receiving mood stabilizers

\begin{tabular}{|c|c|c|c|c|}
\hline Variable & Lithium ( $n=1,317)$ & Valproic acid $(\mathrm{n}=808)$ & Carbamazepine $(n=136)$ & Lamotrigine $(n=665)$ \\
\hline Age (yr) & $51.7 \pm 13.7$ & $50.8 \pm 13.2$ & $52.6 \pm 12.5$ & $46.9 \pm 12.5$ \\
\hline Sex $($ male $=1$, female $=2)$ & $1.5 \pm 0.50$ & $1.53 \pm 0.50$ & $1.5 \pm 0.50$ & $1.54 \pm 0.499$ \\
\hline Height $(\mathrm{cm})$ & $163 \pm 8.7$ & $162 \pm 8.4$ & $162 \pm 8.8$ & $163 \pm 8.4$ \\
\hline Weight (kg) & $63 \pm 12.6$ & $64 \pm 13.2$ & $63 \pm 13.5$ & $62 \pm 12.3$ \\
\hline Education $^{\mathrm{a}}$ & $2.9 \pm 1.1$ & $2.7 \pm 1.1$ & $2.6 \pm 1.1$ & $3.0 \pm 1.1$ \\
\hline Current status of depression $^{b}$ & $0.57 \pm 0.70$ & $0.64 \pm 0.72$ & $0.66 \pm 0.70$ & $0.77 \pm 0.77$ \\
\hline Current status of mania $^{b}$ & $0.20 \pm 0.46$ & $0.25 \pm 0.50$ & $0.33 \pm 0.58$ & $0.18 \pm 0.43$ \\
\hline Current status of psychotic feature ${ }^{b}$ & $0.06 \pm 0.26$ & $0.07 \pm 0.28$ & $0.16 \pm 0.39$ & $0.09 \pm 0.30$ \\
\hline Current status of suicidal ideation ${ }^{\text {b }}$ & $0.09 \pm 0.31$ & $0.13 \pm 0.38$ & $0.17 \pm 0.40$ & $0.16 \pm 0.41$ \\
\hline Current status of substance use ${ }^{b}$ & $0.05 \pm 0.23$ & $0.06 \pm 0.27$ & $0.10 \pm 0.32$ & $0.06 \pm 0.26$ \\
\hline Current status of rapid cycler ${ }^{b}$ & $0.12 \pm 0.33$ & $0.13 \pm 0.34$ & $0.20 \pm 0.40$ & $0.14 \pm 0.34$ \\
\hline Global assessment of functioning $^{c}$ & $0.88 \pm 0.78$ & $0.97 \pm 0.78$ & $1.07 \pm 0.79$ & $0.98 \pm 0.78$ \\
\hline Total number of episode & $8.5 \pm 6.2$ & $8.1 \pm 6.1$ & $11.0 \pm 7.0$ & $7.0 \pm 5.6$ \\
\hline Number of mood stabilizers & $1.4 \pm 0.55$ & $1.5 \pm 0.58$ & $1.8 \pm 0.70$ & $1.4 \pm 0.58$ \\
\hline Number of antidepressants & $0.45 \pm 0.65$ & $0.46 \pm 0.66$ & $0.46 \pm 0.74$ & $0.46 \pm 0.69$ \\
\hline Number of antipsychotics & $0.61 \pm 0.70$ & $0.67 \pm 0.74$ & $0.76 \pm 0.77$ & $0.71 \pm 0.69$ \\
\hline Number of anxiolytics & $0.39 \pm 0.57$ & $0.39 \pm 0.56$ & $0.42 \pm 0.58$ & $0.42 \pm 0.60$ \\
\hline Number of hypnotics & $0.85 \pm 0.82$ & $0.84 \pm 0.80$ & $0.99 \pm 0.85$ & $0.89 \pm 0.87$ \\
\hline Daily dosage (mg/day) & $559 \pm 245$ & $519 \pm 270$ & $363 \pm 197$ & $159 \pm 100$ \\
\hline Range (mg/day) & $25-1,500$ & $100-1,600$ & $50-1,000$ & $5-600$ \\
\hline \multicolumn{5}{|l|}{ Co-administered dosage (mg) } \\
\hline Lithium & - & $579 \pm 249, n=266$ & $616 \pm 255, n=61$ & $649 \pm 260, n=198$ \\
\hline Valproic acid & $538 \pm 267, \mathrm{n}=266$ & - & $677 \pm 273, n=31$ & $552 \pm 285, \mathrm{n}=77$ \\
\hline Carbamazepine & $406 \pm 192, n=61$ & $340 \pm 183, n=31$ & - & $268 \pm 194, n=19$ \\
\hline Lamotrigine & $177 \pm 103, n=198$ & $111 \pm 68, n=77$ & $234 \pm 119, n=19$ & - \\
\hline
\end{tabular}

Values are presented as mean \pm standard deviation.

${ }^{\mathrm{a} J u n i o r}$ high school graduate $=1$, high school $=2$, junior college $=3$, college $=4$, postgraduate degree $=5$. ${ }^{\mathrm{b}}$ Presence $=1$, absent $=0 .{ }^{\mathrm{c}} \mathrm{Global}$ assessment of functioning (GAF) $(81-100)=0, \operatorname{GAF}(61-80)=1, \operatorname{GAF}(41-60)=2, \operatorname{GAF}(<41)=3$.

\section{Statistical Analysis}

The factors associated with the dose of mood stabilizers among bipolar disorder patients were examined using linear regression with forced entry. These factors included sex, height, weight, educational level, GAF score, psychiatric comorbidity, rapid cycle status, substance abuse, mood status, and total number of episodes. The dummy variables included were as follows: male $=1$ and female $=$ 2 ; junior high school graduate $=1$, high school $=2$, junior college $=3$, college $=4$, and postgraduate degree $=5$; $\operatorname{GAF}(81-100)=0, \operatorname{GAF}(61-80)=1, \operatorname{GAF}(41-60)=2$, and GAF $(<41)=3$; depression $=1$ and without depression $=0 ;$ mania $=1$ and without mania $=0 ;$ psychotic feature $=1$ and without psychotic feature $=0$; suicidal ideation $=1$ and without suicidal ideation $=0$; rapid cycler $=$ 1 and not rapid cycler $=0$; and substance abuse $=1$ and without substance abuse $=0$. Pearson correlations were performed for association between doses of mood stabilizers. All statistical tests were based on a two-sided significance level of 0.05. The SPSS Statistics software program for
Windows, version 25.0 (IBM Japan, Tokyo, Japan), was used for all analyses.

\section{RESULTS}

Completed questionnaires on 3,213 outpatients with bipolar disorder were returned from 176 originally solicited outpatient facilities. The characteristics of the subjects are shown in Table 1. Most patients received mood stabilizers such as lithium ( $n=1,317)$, VPA $(n=808)$, CBZ ( $n=136)$, and LTG $(n=665)$. The dose of LTG in patients receiving VPA was significantly lower than that in patients receiving lithium and CBZ.

The dose of lithium was directly correlated with body weight and number of episodes, and GAF and was inversely correlated with age and depression (Table 2). The dose of VPA was correlated with body weight, number of episodes, presence of a rapid cycle and GAF (Table 2). The dose of CBZ was correlated with mania and was inversely correlated with age and the presence of a rapid cy- 
Table 2. Partial correlation coefficients of dose of mood stabilizers prescribed in the outpatients clinics using multiple regression analysis

\begin{tabular}{|c|c|c|c|c|}
\hline Variable & Lithium $(n=1,317)$ & Valproic acid $(n=809)$ & Carbamazepine $(n=136)$ & Lamotrigine $(n=665)$ \\
\hline Age & $-0.186^{* * *}$ & -0.038 & $-0.206^{*}$ & -0.003 \\
\hline Sex $($ male $=1$, female $=2)$ & -0.047 & 0.004 & 0.076 & 0.042 \\
\hline Height & 0.077 & -0.017 & 0.163 & 0.062 \\
\hline Weight & $0.115^{* *}$ & $0.103 *$ & -0.125 & -0.003 \\
\hline Education periods ${ }^{\mathrm{a}}$ & 0.045 & 0.055 & 0.193 & 0.007 \\
\hline Number of episode & $0.073^{* *}$ & $0.116^{* *}$ & 0.049 & $0.084 *$ \\
\hline Presence of depression ${ }^{b}$ & $-0.070^{*}$ & -0.057 & 0.017 & $-0.113^{*}$ \\
\hline Presence of mania ${ }^{b}$ & 0.021 & 0.042 & $0.237^{*}$ & $-0.082^{*}$ \\
\hline Presence of psychotic features ${ }^{b}$ & 0.012 & -0.018 & 0.095 & $0.089 *$ \\
\hline Presence of suicidal ideation ${ }^{b}$ & -0.031 & 0.026 & -0.047 & 0.015 \\
\hline Presence of substance use $\mathrm{e}^{\mathrm{b}}$ & -0.032 & -0.015 & -0.139 & -0.005 \\
\hline Presence of rapid cycler ${ }^{\mathrm{b}}$ & 0.019 & $0.090^{* *}$ & $-0.203^{*}$ & $0.101^{*}$ \\
\hline Global assessment of functioning ${ }^{\mathrm{c}}$ & $0.108^{* *}$ & $0.072^{*}$ & -0.011 & 0.061 \\
\hline $\mathrm{R}$ & $0.328^{* * *}$ & $0.213^{* * *}$ & $0.450^{* *}$ & $0.194 *$ \\
\hline
\end{tabular}

$\mathrm{R}$, multiple correlation coefficients.

Junior high school graduate $=1$, high school $=2$, junior college $=3$, college $=4$, postgraduate degree $=5$. ${ }^{\mathrm{b}}$ Presence $=1$, absent $=0$. ${ }^{\mathrm{c}} \mathrm{Global}$ assessment of functioning (GAF) $(81-100)=0, \mathrm{GAF}(61-80)=1, \mathrm{GAF}(41-60)=2, \mathrm{GAF}(<41)=3$.

${ }^{*} p<0.05,{ }^{* *} p<0.01,{ }^{* * *} p<0.001$.

Table 3. Correlations between doses of mood stabilizers co-administered

\begin{tabular}{lllll}
\hline \multicolumn{1}{c}{ Drugs } & Lithium $(\mathrm{n}=1,317)$ & Valproic acid $(\mathrm{n}=808)$ & Carbamazepine $(\mathrm{n}=136)$ & Lamotrigine $(\mathrm{n}=665)$ \\
\hline Valproic acid & $0.254^{* * *}(\mathrm{n}=266)$ & & \\
Carbamazepine & $0.346^{* *}(\mathrm{n}=61)$ & $0.454^{* *}(\mathrm{n}=31)$ & \\
Lamotrigine & $0.139^{*}(\mathrm{n}=198)$ & $-0.038(\mathrm{n}=77)$ & $0.557^{* *}(\mathrm{n}=19)$ & \\
\hline
\end{tabular}

${ }^{*} p<0.05,{ }^{* *} p<0.01,{ }^{* * *} p<0.001$.

cle (Table 2). The dose of LTG was correlated with the number of episodes, psychotic features, and the presence of a rapid cycle and was inversely correlated with the presence of depression and mania (Table 2).

The doses of coadministered mood stabilizers were significantly correlated with the combination of VPA and LTG (Table 3).

\section{DISCUSSION}

Mood stabilizers such as lithium and anticonvulsants are still standard-of-care for the acute and long-term treatment of bipolar disorder. The results of this study showed that age, body weight, number of episodes, presence of current mood disorders, presence of rapid cycle and social adjustment are associated with the doses of mood stabilizers, and this is the first report regarding mood stabilizer dosing and its related factors in the world.

We found a significant inverse correlation between daily doses of lithium and CBZ and age. These findings are reasonable because lithium is excreted by the kidneys
[12], and renal function decreases with age [13]. In addition, CBZ is metabolized by liver enzymes, particularly CYP3A4 [12], whose activity decreases with age [14]. Therefore, serum concentrations of lithium and CBZ tend to increase with age. On the other hand, VPA and LTG are metabolized by glucuronic acid conjugation, which, unlike compared with CYPs, is not influenced by age.

Depression decreased the doses of lithium and LTG in the study. The recommended doses of lithium were 600$1,200 \mathrm{mg} /$ day during the manic episode and $600-1,800$ $\mathrm{mg} /$ day during the depressive episode [3], suggesting that the daily dose of lithium during the depressive episode should be higher than that during the manic episode. However, our findings demonstrated a lower dose of lithium in patients with depression, although the average daily dose of lithium was approximately 560 mg/day, which is low compared with the world standard recommended dose [3]. We do not have a clear explanation for this discrepancy. The recommended doses of LTG were $50-$ $200 \mathrm{mg} /$ day during depressive episodes and 50-400 $\mathrm{mg} /$ day during maintenance episodes [3], suggesting that 
the daily dose of LTG during depressive episodes should be lower than that during maintenance episodes. Our results for LTG were in line with the guidelines [3].

The number of episodes was correlated with the doses of lithium and VPA in the study. Although the recommended doses for these mood stabilizers were not shown in the guidelines, lithium and VPA were consistently ranked as the 1st line treatments [3-5]. Therefore, lithium and VPA might be regarded as the main agents for the prevention of repeated episodes.

The presence of rapid cycling was directly correlated with the doses of VPA and LTG and inversely correlated with the dose of CBZ. CINP guidelines say that aripiprazole, quetiapine and VPA were the first-line treatments for rapid-cycling patients, and olanzapine and lithium were recommended as second-line strategies [15], but LTG was not different from placebo in the context of maintenance treatment in patients with rapid cycling [16]. On the other hand, a combination of LTG and mood stabilizers (or atypical antipsychotics) was potentially preferable during episodes of current depression according to KMAP-BP 2018 [5].

Patients who have better response to lithium are a group of patients with bipolar disorder core phenotype clinical features $[17,18]$. An non-episodic pattern of the mania-depression interval, high rates of comorbid conditions, rapid cycling and younger age at illness onset have been identified as potentially risk against recurrence during the lithium treatment [19-22]. Patients who have better response to VPA are characterized by the presence of pure, mixed or dysphoric mania. In addition, elder age at onset, absence of rapid cycling, absence of concurrent substance abuse and lack of response or intolerance to lithium are clinical predictors of positive outcome of treatment with VPA [23]. Patients who have better response to CBZ seems to be predicted by clinical features such as mood-incongruent psychosis and lack of response or intolerance to lithium, while contrasting findings are potentially predictive of presence of rapid cycling, severe mania and dysphoric mania [24,25]. Earlier onset of symptoms, nonepisodic course of illness, rapid cycling, COmorbidity with a panic or substance use disorder, fewer hospitalizations, fewer prior medication trials and male sex may be predictors in patients who have better response to LTG $[26,27]$.

This study has several notable limitations. First, this was a cross-sectional study, so we could not determine causal relationships. The second limitation of this study was the lack of a structural clinical evaluation of patients, although clinical evaluation in this study was performed by doctors in charge of the patients who were well trained. Additionally, selection bias may have occurred because the subjects were not randomized. Finally, there may be problems with population heterogeneity because we did not distinguish between bipolar I and II. Thus, some interrater variability cannot be excluded. Further longitudinal studies are needed to confirm the detailed prescription patterns for patients with bipolar disorder in Japan.

\section{- Acknowledgments}

This study was supported by Ken Tanaka memorial research grant.

The authors thank the following psychiatrists belonging to the Japanese Association of Neuro-Psychiatric Clinics: Dr. Toshihiko Lee, Dr. Norio Okamoto, Dr. Makoto Nakamura, Dr. Junkou Sato, Dr. Kazunori Otaka, Dr. Satoshi Terada, Dr. Tadashi Ito, Dr. Munehide Tani, Dr. Atsushi Satomura, Dr. Hiroshi Sato, Dr. Hideki Nakano, Dr. Yoichi Nakaniwa, Dr. Eiichi Hirayama, Dr. Keiichi Kobatake, Dr. Koji Tanaka, Dr. Mariko Watanabe, Dr. Shiguyuki Uehata, Dr. Asana Yuki, Dr. Nobuko Akagaki, Dr. Michie Sakano, Dr. Akira Matsukubo, Dr. Yukihisa Kibota, Dr. Yasuyuki Inada, Dr. Hiroshi Oyu, Dr. Tsuneo Tsubaki, Dr. Tatsuji Tamura, Dr. Shigeki Akiu, Dr. Atsuhiro Kikuchi, Dr. Keiji Sato, Dr. Kazuyuki Fujita, Dr. Fumio Handa, Dr. Hiroyuki Karasawa, Dr. Kazuhiro Nakano, Dr. Kazuhiro Omori, Dr. Seiji Tagawa, Dr. Daisuke Maruno, Dr. Hiroaki Furui, Dr. You Suzuki, Dr. Takeshi Fujita, Dr. Yukimitsu Hoshino, Dr. Kikuko Ota, Dr. Akira Itami, Dr. Kenichi Goto, Dr. Yoshiaki Yamano, Dr. Kiichiro Koshimune, Dr. Junko Matsushita, Dr. Takatsugu Nakayama, Dr. Kazuyoshi Takamuki, Dr. Nobumichi Sakamoto, Dr. Miho Shimizu, Dr. Muneo Shimura, Dr. Norio Kawase, Dr. Ryouhei Takeda, Dr. Takuya Hirota, Dr. Hideko Fujii, Dr. Yoichiro Watanabe, Dr. Riichiro Narabayashi, Dr. Yutaka Fujiwara, Dr. Kazu Kobayashi, Dr. Yuko Urabe, Dr. Miyako Oguru, Dr. Osamu Miura, Dr. Yoshio Ikeda, Dr. Hidemi Sakamoto, Dr. Yosuke Yonezawa, Dr. Yoichi Takei, Dr. Toshimasa Sakane, Dr. Kiyoshi Oka, Dr. Kyoko Tsuda, Dr. Shigemitsu Hayashi, Dr. Kunihiko Kawamura, Dr. Yasushi Furuta, Dr. Kazuko Miyauchi, Dr. Yoshio Miyauchi, Dr. Mikako 
Oyama, Dr. Keizo Hara, Dr. Misako Sakamoto, Dr. Shigeki Masumoto, Dr. Yasuhiro Kaneda, Dr. Yoshiko Kanbe, Dr. Masayuki Iwai, Dr. Naohisa Waseda, Dr. Nobuhiko Ota, Dr. Takahiro Hiroe, Dr. Ippei Ishii, Dr. Hideki Koyama, Dr. Terunobu Otani, Dr. Osamu Takatsu, Dr. Takashi Ito, Dr. Norihiro Marui, Dr. Toru Takahashi, Dr. Tetsuro Oomori, Dr. Toshihiko Fukuchi, Dr. Kazumichi Egashira, Dr. Kiyoshi Kaminishi, Dr. Ryuichi Iwata, Dr. Satoshi Kawaguchi, Dr. Yoshinori Morimoto, Dr. Hirohisa Endo, Dr. Yasuo Imai, Dr. Eri Kohno, Dr. Aki Yamamoto, Dr. Naomi Hasegawa, Dr. Sadamu Toki, Dr. Hideyo Yamada, Dr. Hiroyuki Taguchi, Dr. Hiroshi Yamaguchi, Dr. Hiroki Ishikawa, Dr. Sakura Abe, Dr. Kazuhiro Uenoyama, Dr. Kazunori Koike, Dr. Yoshiko Kamekawa, Dr. Michihito Matsushima, Dr. Ken Ueki, Dr. Sintaro Watanabe, Dr. Tomohide Igata, Dr. Yoshiaki Higashitani, Dr. Eiichi Kitamura, Dr. Junko Sanada, Dr. Takanobu Sasaki, Dr. Kazuko Eto, Dr. Ichiro Nasu, Dr. Kenichiro Sinkawa, Dr. Yukio Oga, Dr. Michio Tabuchi, Dr. Daisuke Tsujimura, Dr. Tokunai Kataoka, Dr. Kyohei Noda, Dr. Nobuhiko Imato, Dr. Ikuko Nitta, Dr. Yoshihiro Maruta, Dr. Satoshi Seura, Dr. Toru Okumura, Dr. Osamu Kino, Dr. Tomoko Ito, Dr. Ryuichi Iwata, Dr. Wataru Konno, Dr. Toshio Nakahara, Dr. Masao Nakahara, Dr. Hiroshi Yamamura, Dr. Masatoshi Teraoka, Dr. Masato Nishio, Dr. Miwa Mochizuki, Dr. Tsuneo Saitoh, Dr. Tetsuharu Kikuchi, Dr. Chika Higa, Dr. Hiroshi Sasa, Dr. Yuichi Inoue, Dr. Muneyoshi Yamada, Dr. Yoko Fujioka, Dr. Kuniaki Maekubo, Dr. Hiroaki Jitsuiki, Dr. Toshihito Tsutsumi, Dr. Yasumasa Asanobu, Dr. Seiji Inomata, Dr. Kazuhiro Kodama, Dr. Aikihiro Takai, Dr. Asako Sanae, Dr. Shinichiro Sakurai, Dr. Kazuhide Tanaka, Dr. Masahiko Shido, Dr. Haruhisa Ono, Dr. Wataru Miura, Dr. Yukari Horie, Dr. Tetso Tashiro, Dr. Tomohide Mizuno, Dr. Naohiro Fujikawa, Dr. Hiroshi Terada, Dr. Kenji Taki, Dr. Kyoko Kyotani, Dr. Masataka Hatakoshi, Dr. Katsumi Ikeshita, Dr. Keiji Kaneta, Dr. Ritsu Shikiba, Dr. Tsuyoshi lijima, Dr. Masaru Yoshimura, Dr. Masumi Ito, Dr. Shunsuke Murata, Dr. Mio Mori, and Dr. Toshio Yokouchi.

\section{a Conflicts of Interest}

Dr. Yasui-Furukori has received grant/research support or honoraria from and been on the speakers of Dainippon-Sumitomo Pharma, Mochida Pharmaceutical, MSD, and Otsuka Pharmaceutical. Dr. Shimoda has re- ceived research support from Novartis Pharma, Dainippon Sumitomo Pharma, Astellas Pharma, Meiji Seika Pharma, Eisai, Pfizer, Otsuka Pharmaceutical, Daiichi Sankyo, and Takeda Pharmaceutical, and honoraria from Eisai, Mitsubishi Tanabe Pharma, Takeda Pharmaceutical, Meiji Seika Pharma, Janssen Pharmaceutical, Shionogi, Dainippon Sumitomo Pharma, Daiichi Sankyo, and Pfizer. Dr. Yoshimura has received speaker's honoraria from Eli Lilly, Janssen, Dainippon Sumitomo, Otsuka, Meiji, Pfizer and Shionogi. Dr. Kato has received grant funding from Japan Society for the Promotion of Science, SENSHIN Medical Research Foundation and Japan Research Foundation for Clinical Pharmacology, and speaker's honoraria from Dainippon-Sumitomo Pharma, Otsuka, Meiji-Seika Pharma, Eli Lilly, MSD K.K., GlaxoSmithkline, Pfizer, Janssen Pharmaceutical, Shionogi, Mitsubishi Tanabe Pharma, Takeda Pharmaceutical and Ono Pharmaceutical. Dr. Azekawa has received speaker's honoraria from Eli Lilly, Otsuka Pharmaceutical, and Pfizer. Dr. Ueda has received manuscript fees or speaker's honoraria from Eli Lilly, Janssen Pharmaceutical, Meiji Seika Pharma, Mitsubishi Tanabe Pharma, MSD, Otsuka Pharmaceutical, Pfizer, Sumitomo Dainippon Pharma, Takeda Pharmaceutical, and Yoshitomi Yakuhin. Dr. Edagawa has received speaker's honoraria from Eli Lilly, Meiji Seika Pharma, Mitsubishi Tanabe Pharma, MSD, Otsuka Pharmaceutical, Pfizer, Sumitomo Dainippon Pharma, Kyowa and Yoshitomi Yakuhin. Dr. Katsumoto has received speaker's honoraria from Daiichi Sankyo, Eisai, Eli Lilly, Janssen Pharmaceutical, Kyowa Pharmaceutical, Meiji Seika Pharma, Mitsubishi Tanabe Pharma, MSD, Otsuka Pharmaceutical, Pfizer, Sumitomo Dainippon Pharma, UCB. Dr. Kubota has received consultant fees from Pfizer and Meiji-Seika Pharma and speaker's honoraria from Meiji-Seika Pharma, Eli Lilly, Janssen Pharmaceutical, Dainippon Sumitomo Pharma, Mitsubishi Tanabe Pharma, Yoshitomi Yakuhin, Otsuka Pharmaceutical, and Eisai. Dr. Goto has received manuscript fees or speaker's honoraria from Eli Lilly, Janssen Pharmaceutical, Meiji Seika Pharma, Mitsubishi Tanabe Pharma, MSD, Otsuka Pharmaceutical, Sumitomo Dainippon Pharma. Dr. Hongo has received manuscript fees or speaker's honoraria from Eli Lilly, Janssen Pharmaceutical, Kyowa Pharmaceutical, Meiji Seika Pharma, Mitsubishi Tanabe Pharma, Otsuka Pharmaceutical, Pfizer, Shionogi, Sumitomo Dainippon Pharma, and Yoshitomi Yakuhin. Dr. Tsuboi has received consultant fees from Pfizer and 
speaker's honoraria from Eli Lilly, Meiji-Seika Pharma, MSD, Janssen Pharmaceutical, Dainippon Sumitomo Pharma, Mitsubishi Tanabe Pharma, Yoshitomi Yakuhin, Mochida Pharmaceutical, Otsuka Pharmaceutical, Kyowa Pharmaceutical, and Takeda Pharmaceutical. Dr. Nakagawa has received lecture fees from Pfizer, Eli Lilly, Otsuka, Janssen Pharmaceutical, Mitsubishi Tanabe, Mochida, Dainippon Sumitomo and NTT Docomo, and participated in an advisory board for Takeda, Meiji Seika and Tsumura. Dr. Kikuchi has received consultant fees from Takeda Pharmaceutical and Center for Cognitive Behavioral Therapy and Training. Dr. Watanabe has received manuscript fees or speaker's honoraria from Daiichi Sankyo, Eisai, Eli Lilly, GlaxoSmithKline, Janssen Pharmaceutical, Kyowa Pharmaceutical, Meiji Seika Pharma, Mitsubishi Tanabe Pharma, MSD, Otsuka Pharmaceutical, Pfizer, Shionogi, Sumitomo Dainippon Pharma, Takeda Pharmaceutical, Yoshitomi Yakuhin, and has received research/ grant support from Astellas Pharma, Daiichi Sankyo, Eisai, MSD, Mitsubishi Tanabe Pharma, Meiji Seika Pharma, Otsuka Pharmaceutical, Pfizer, Shionogi, Sumitomo Dainippon Pharma, and is a consultant of Eisai, Eli Lilly, Kyowa Pharmaceutical, Otsuka Pharmaceutical, Pfizer, Sumitomo Dainippon Pharma, Taisho Toyama Pharmaceutical, and Takeda Pharmaceutical.

\section{a Author Contributions}

Conceptualization: Norio Yasui-Furukori and Koichiro Watanabe. Data acquisition: Naoto Adachi, Yukihisa Kubota, Takaharu Azekawa, Eiichiro Goto, Koji Edagawa, Eiichi Katsumoto, Seiji Hongo, Hitoshi Ueda, and Kazuhira Miki. Formal analysis: Naoto Adachi. Funding: Koichiro Watanabe. Supervision: Masaki Kato, Reiji Yoshimura, Atsuo Nakagawa, Toshiaki Kikuchi, Takashi Tsuboi, and Koichiro Watanabe. Writing - original draft: Norio YasuiFurukori. Writing - review \& editing: Norio Yasui-Furukori and Kazutaka Shimoda.

ORCID

Norio Yasui-Furukori

https://orcid.org/0000-0002-4414-3770

Naoto Adachi https://orcid.org/0000-0001-8376-4900

Yukihisa Kubota https://orcid.org/0000-0002-9837-6799

Takaharu Azekawa https://orcid.org/0000-0003-4294-4600

Eiichiro Goto https://orcid.org/0000-0001-8087-4549

Koji Edagawa https://orcid.org/0000-0003-1970-0042
Eiichi Katsumoto https://orcid.org/0000-0001-9417-4391 Seiji Hongo https://orcid.org/0000-0002-5997-8906 Hitoshi Ueda https://orcid.org/0000-0001-8950-2872 Kazuhira Miki https://orcid.org/0000-0003-3056-8038 Masaki Kato https://orcid.org/0000-0001-6727-7272 Reiji Yoshimura https://orcid.org/0000-0002-7637-5576 Atsuo Nakagawa https://orcid.org/0000-0002-2294-2571 Toshiaki Kikuchi https://orcid.org/0000-0003-1025-8796 Takashi Tsuboi https://orcid.org/0000-0002-8253-8888 Koichiro Watanabe https://orcid.org/0000-0002-0736-7070 Kazutaka Shimoda https://orcid.org/0000-0002-9663-6243

\section{REFERENCES}

1. Parker GB, Graham RK, Tavella G. Is there consensus across international evidence-based guidelines for the management of bipolar disorder? Acta Psychiatr Scand 2017;135:515-526.

2. Miura T, Noma H, Furukawa TA, Mitsuyasu H, Tanaka S, Stockton S, et al. Comparative efficacy and tolerability of pharmacological treatments in the maintenance treatment of bipolar disorder: a systematic review and network metaanalysis. Lancet Psychiatry 2014;1:351-359.

3. Fountoulakis KN, Grunze H, Vieta E, Young A, Yatham L, Blier P, et al. The International College of Neuro-Psychopharmacology (CINP) treatment guidelines for bipolar disorder in adults (CINP-BD-2017), part 3: the clinical guidelines. Int ) Neuropsychopharmacol 2017;20:180-195.

4. Yatham LN, Kennedy SH, Parikh SV, Schaffer A, Bond DJ, Frey $\mathrm{BN}$, et al. Canadian Network for Mood and Anxiety Treatments (CANMAT) and International Society for Bipolar Disorders (ISBD) 2018 guidelines for the management of patients with bipolar disorder. Bipolar Disord 2018;20:97-170.

5. Woo YS, Bahk WM, Lee JG, Jeong JH, Kim MD, Sohn I, et al. Korean Medication Algorithm Project for Bipolar Disorder 2018 (KMAP-BP 2018): fourth revision. Clin Psychopharmacol Neurosci 2018;16:434-448.

6. Hiemke C, Bergemann N, Clement HW, Conca A, Deckert J, Domschke K, et al. Consensus guidelines for therapeutic drug monitoring in neuropsychopharmacology: update 2017. Pharmacopsychiatry 2018;51:e1.

7. Steen NE, Aas M, Simonsen C, Dieset I, Tesli M, Nerhus M, et al. Serum concentrations of mood stabilizers are associated with memory, but not other cognitive domains in psychosis spectrum disorders; explorative analyses in a naturalistic setting. Int J Bipolar Disord 2016;4:24.

8. Pharmaceuticals and Medical Devices Agency. Regarding compliance with blood concentration measurement during administration of lithium carbonate; request of the pharmaceutical products proper use from Pharmaceuticals and Medical Devices Agency (PMDA) [Internet]. Tokyo: Pharmaceuticals and Medical Devices Agency; 2012 Sep [cited at 2020 May 1]. Available from: https://www.pmda.go.jp/files/000145551. 
pdf\#search=\%27\%E3\%83\%AA\%E3\%83\%81\%E3\%82\%A6 $\%$ \%3\%83\%AO+\%E8\%A1\%80\%E4\%B8\%AD\%E6\%BF\%83 $\% E 5 \% B A \% A 6+\% E 9 \% A 0 \% B B \% E 5 \% B A \% A 6 \% 27$. Japanese.

9. Murru A, Popovic D, Pacchiarotti I, Hidalgo D, LeónCaballero J, Vieta E. Management of adverse effects of mood stabilizers. Curr Psychiatry Rep 2015;17:603.

10. Adachi N, Kubota Y, Goto E, Ueda H, Azekawa T, Hongo S, et al. [Estimated population with affective disorders in membership clinics of the Japanese Association of NeuroPsychiatric Clinics]. J Jpn Assoc Psychiatr Clin 2016;42:957959. Japanese.

11. Tsuboi T, Suzuki T, Azekawa T, Adachi N, Ueda H, Edagawa $\mathrm{K}$, et al. Factors associated with non-remission in bipolar disorder: the multicenter treatment survey for bipolar disorder in psychiatric outpatient clinics (MUSUBI). Neuropsychiatr Dis Treat 2020;16:881-890.

12. Wang PW, Ketter TA. Pharmacokinetics of mood stabilizers and new anticonvulsants. Psychopharmacol Bull 2002;36: 44-66.

13. Williams L, Lowenthal DT. Drug therapy in the elderly. South Med 1992;85:127-131.

14. Greenblatt DJ, Harmatz JS, Shapiro L, Engelhardt N, Gouthro TA, Shader RI. Sensitivity to triazolam in the elderly. NEngl J Med 1991;324:1691-1698.

15. Fountoulakis KN, Kontis D, Gonda X, Yatham LN. A systematic review of the evidence on the treatment of rapid cycling bipolar disorder. Bipolar Disord 2013;15:115-137.

16. Calabrese JR, Suppes T, Bowden CL, Sachs GS, Swann AC, McElroy SL, et al. A double-blind, placebo-controlled, prophylaxis study of lamotrigine in rapid-cycling bipolar disorder. J Clin Psychiatry 2000;61:841-850.

17. Baldessarini RJ, Tondo L. Does lithium treatment still work? Evidence of stable responses over three decades. Arch Gen
Psychiatry 2000;57:187-190.

18. Soares JC, Gershon S. The psychopharmacologic specificity of the lithium ion: origins and trajectory. J Clin Psychiatry 2000;61 Suppl 9:16-22.

19. Grof $\mathrm{P}, \mathrm{Alda}$ M, Grof $\mathrm{E}$, Fox D, Cameron P. The challenge of predicting response to stabilising lithium treatment. The importance of patient selection. Br J Psychiatry Suppl 1993;(21): 16-19.

20. Calabrese JR, Fatemi SH, Kujawa M, Woyshville MJ. Predictors of response to mood stabilizers. J Clin Psychopharmacol 1996;16(2 Suppl 1):24S-31S.

21. Bowden CL. Clinical correlates of therapeutic response in bipolar disorder. J Affect Disord 2001;67:257-265.

22. Kleindienst N, Engel R, Greil W. Which clinical factors predict response to prophylactic lithium? A systematic review for bipolar disorders. Bipolar Disord 2005;7:404-417.

23. Ketter TA, Wang PW. Predictors of treatment response in bipolar disorders: evidence from clinical and brain imaging studies. J Clin Psychiatry 2002;63 Suppl 3:21-25.

24. Alda M. The phenotypic spectra of bipolar disorder. Eur Neuropsychopharmacol 2004;14 Suppl 2:S94-S99.

25. De León OA. Antiepileptic drugs for the acute and maintenance treatment of bipolar disorder. Harv Rev Psychiatry 2001;9:209-222.

26. Obrocea GV, Dunn RM, Frye MA, Ketter TA, Luckenbaugh DA, Leverich GS, et al. Clinical predictors of response to lamotrigine and gabapentin monotherapy in refractory affective disorders. Biol Psychiatry 2002;51:253-260.

27. Passmore MJ, Garnham J, Duffy A, MacDougall M, Munro A, Slaney $\mathrm{C}$, et al. Phenotypic spectra of bipolar disorder in responders to lithium versus lamotrigine. Bipolar Disord 2003;5: 110-114. 